\title{
Interés y motivación del estudiantado de Educación Secundaria en el uso de Aurasma en el aula de Educación Física \\ Secondary Education students' interest and motivation towards using Aurasma in Physical Education classes

\author{
*Antonio José Moreno Guerrero, ${ }^{*}$ Carmen Rodríguez Jiménez, *Magdalena Ramos Navas-Parejo, **Jose María Sola \\ Reche \\ * Universidad de Granada (España), **Universidad de Alicante (España)
}

Resumen. Las tecnologías de la información y la comunicación (TIC) están inmersas en los procesos de enseñanza y aprendizaje. El objetivo de esta investigación es conocer la predisposición y motivación en el aprendizaje de la orientación espacial de los alumnos de Educación Secundaria Obligatoria (ESO), a través del uso de la aplicación Aurasma. La metodología de investigación es cuantitativa, descriptiva, correlacional y predictiva. Los instrumentos utilizados son el FEFS-J y el cuestionario EDMCQ-PE. La muestra está formada por 1076 alumnos de la ESO de Andalucía. Los resultados muestran que existe una relación entre la valoración dada por los alumnos en relación con el uso de Aurasma, con el interés mostrado hacia la tarea y su motivación, teniendo una influencia directa y consistente en la energía dada por la asignatura de Educación Física para el desarrollo de otras tareas. Se concluye que el uso de Aurasma en las sesiones de Educación Física para el desarrollo de la orientación espacial es valorado positivamente por los alumnos de la ESO, mostrando interés y motivación por la asignatura.

Palabras Clave: educación física, realidad aumentada, aurasma, educación secundaria, motivación.

\begin{abstract}
Information and communication technology (ICT) is immersed into teaching and learning processes. The objective of this research is to know the predisposition and motivation towards learning spatial orientation in students of Compulsory Secondary Education, through the use of the Aurasma application. The research methodology is quantitative, descriptive, correlational, and predictive. The instruments used are FEFS-J and EDMCQ-PE questionnaires. The sample was composed by 1076 students from Compulsory Secondary Education in Andalusia. The results show that there is a relationship between the assessment given by the students in relation to the use of Aurasma during the session, and interest and motivation shown towards the task, with direct and consistent influence on the energy put in the subject of Physical Education for the development of other tasks. As a conclusion, the use of Aurasma for the development of spatial orientation in Physical Education classes is valued positively by the students of Compulsory Secondary Education, showing interest and motivation towards the subject.
\end{abstract}

Keywords: physical education, augmented reality, aurasma, secondary education, active methodologies, motivation.

\section{Introducción}

El uso de la tecnología en el ámbito educativo se encuentra hoy en día en pleno auge, debido a los últimos avances tecnopedagógicos enfocados en incluir las tecnologías de la información y comunicación (TIC) en los procesos de enseñanza y aprendizaje, con el objetivo de formar entornos activos de aprendizaje (Gómez-Gonzalvo, Molina-Alventosa \& Devís, 2018; Rodríguez, Cáceres \&Alonso, 2018). Dado el carácter ubicuo y ergonómico que poseen este tipo de herramientas pedagógicas (Fombona y Pascual, 2017), son cada vez más usuales en la docencia (Viñals \& Cuenta, 2016). Este hecho supone para los discentes un nuevo rol, en el que las formas de comunicación y colaboración con los docentes, compañeros y contenidos se realiza de modo digital (Radu, 2014).

A través de las TIC se puede mejorar la participación, motivar y despertar el interés de los estudiantes por su formación (Aznar, Cáceres, Trujillo \& Romero, 2019; Marín y Muñoz, 2018), debido al enriquecimiento e interacción que permite con la información (Villalustre \& Del Moral, 2017). Para el profesorado también supone un cambio radical de su práctica docente (Area, Hernández \& Sosa, 2016; Díaz, Molina-García \& Monfort-Pañego, 2019), convirtiéndose en guía del proceso de enseñanza y aprendizaje (Castañeda,

Fecha recepción: 02-12-19. Fecha de aceptación: 29-01-20

Carmen Rodríguez Jiménez

carmenrj@ugr.es
Esteve \& Adell, 2018), desarrollando además una serie de habilidades tecnopedagógicas para poder hacer uso de las TIC en el aula (Cabero \& Barroso, 2018; Fuentes, López \& Pozo, 2019; Marqués, Cela \& Gisbert, 2017)

Una herramienta tecnopedagógica que se encuentra en pleno apogeo en el ámbito educativo es la realidad aumentada (RA) (Cabero \& Roig, 2019; Rodríguez, Hinojo \& Agreda, 2019), la cual permite apoyar los procesos de enseñanza y aprendizaje, facilitando la comprensión de la materia objeto de estudio (Chen, Liu, Cheng \& Huang, 2017). La RA se define como la innovación capaz de combinar información física y digital en tiempo real, creando una realidad más completa (Barroso, Cabero, García, Calle, Gallego \& Casado, 2017), a través de dispositivos tecnológicos sencillos como el móvil o la tablet (Gómez, Trujillo, Aznar \& Cáceres, 2018). Este recurso tecnopedagógico es adecuado para todas las etapas educativas, desde Educación Infantil (López, Pozo \& López, 2019) hasta Educación Superior (Garay, Tejada \& Castaño, 2017).

Las ventajas que la RA aporta la educación son las siguientes: el discente se convierte en protagonista de su aprendizaje (Cabero, Llorente \& Marín, 2017), se produce un aumento de la motivación (Akcayir \& Akcayir, 2017), mejora la autorregulación (Marín, Cabero \& Gallego, 2018), así como el interés (Cheng, 2017), permite experimentar con materiales y contenidos (Fombona \& Vázquez, 2017), repercute en el desarrollo de la competencia digital (Toledo \& Sánchez, 2017) y promueve el aprendizaje significativo, constructivista, colaborativo, por descubrimiento y ubicuo (Cabero, Llorente 
\& Gutiérrez, 2017). De esta forma se consigue a su vez, mejorar los resultados académicos y el ambiente de aula (Prendes, 2015).

La mayoría de las investigaciones sobre la RA se han basado en cuantificar la producción científica (Lorenzo \& Scagliarini, 2018; Suh \& Prophet, 2018), en las novedades tecnológicas (Muhamedyev, Aliguliyev, Shokishalov \& Mustakayev, 2018) y en las variaciones y proyecciones de esta metodología pedagógica (Fombona, Pascual \& González, 2017), extendiéndose su campo de acción en la ingeniería, las ciencias (Heradio, de la Torre, Galán, Cabrerizo, Herrera \& Dormido, 2016), el ámbito empresarial (Álvarez, Castillo \& Geldes, 2017),y en educación mediante aplicaciones relacionadas con el turismo, el ocio, el marketing y el transporte (Jaramillo, Silva, Adarve, Velásquez, Páramo \& Gómez, 2018).

Para la creación de objetos de RA existe una plataforma gratuita llamada Aurasma creada por la empresa de software Autonomy en 2011, la cual utiliza el reconocimiento avanzado de imágenes y patrones para detectar una imagen de forma muy similar a como lo hace el cerebro humano (Delello, 2014). El contenido interactivo se puede combinar con imágenes, sonidos, videos y animaciones llamadas «auras». Diversas investigaciones muestran que es un recurso motivador, de fácil manejo (Roig.Vila, Lorenzo-Lledó \& Mengual-Andres, 2019), que aumenta el compromiso, el entusiasmo (Delello, 2014), facilita una mejor comprensión, e integra la teoría con la práctica (Opris, Cortinas, Ionescu \& Gogoase, 2018).

El uso de la RA en la materia de educación física facilita la comprensión teórica (Hsiao, 2013), y mejora las habilidades motoras complejas (Chang, Zhang, Huang, Liu \& Sung, 2019; Huang \& Reynoso, 2018), el rendimiento cognitivo, las relaciones sociales (Ruiz-Ariza, Casuso, Suárez-Manzano \& Martínez-López, 2018), las capacidades en orientación espacial y estimación de distancias (Gómez-García, Trujillo-Torres, Aznar-Díaz \& Cáceres-Reche, 2018) y la competencia digital (Gallego-Lema, Muñoz-Cristobal, Arribas-Cubero \& Rubia-Avi, 2017), resultando en este campo una herramienta pedagógica muy valiosa. A los docentes les ayuda a conectar diferentes espacios físicos, apoyando al mismo tiempo las tecnologías y actividades de su práctica diaria, y transfiriendo parte de la carga de la orquestación de los profesores a los estudiantes (Muñoz-Cristobal, Gallego-Lema, ArribasCubero, Martínez-Mones \& Asensio-Pérez, 2017).

\section{Justificación y objetivos del estudio}

No existe una gran producción sobre el uso de la RA en el campo de educación física, tal y como se observa en las bases de datos de Web of Science o Scopus. Por ello, surge este estudio, que tiene como objetivo conocer la predisposición y motivación en el aprendizaje de la orientación espacial del alumnado de la ESO, mediante el uso de Aurasma. A partir de este enunciado se formulan las siguientes cuestiones:

- ¿Cómo valoran los estudiantes el uso de Aurasma en las sesiones de Educación Física?

- ¿Qué interés muestran los estudiantes durante el desarrollo de sesiones en donde se usa la realidad aumentada?

- ¿Qué motivación muestra el alumnado mediante la utilización de la realidad aumentada en los procesos de enseñanza y aprendizaje?
- ¿Qué relación existe entre la valoración del uso de Aurasma y el interés de los estudiantes por el desarrollo de las sesiones de Educación Física?

- ¿Qué relación existe entre la valoración del uso de Aurasma y la motivación del estudiante en el desarrollo de las sesiones de Educación Física?

- ¿Influye la valoración del uso de Aurasma en el gusto por las clases de Educación Física?

- ¿Influye la valoración del uso de Aurasma en la motivación del estudiante en la Educación Física?

\section{Método}

La presente investigación ha sido realizada a través de es un diseño de tipo descriptivo, correlacional y predictivo. Todo ello, dentro de una metodología cuantitativa, atendiendo a las disposiciones establecidas por Hernández, Fernández \& Baptista (2014).

\section{Muestra}

La muestra está formada por estudiantes que cursan la materia de Educación Física en la etapa de la ESO en centros educativos de Andalucía, llevando a cabo un muestreo por conveniencia, debido a que se realizó un llamamiento a diversos centros, para solicitar docentes de Educación Física voluntarios que quisieran participar en la actividad, siendo un total de 26 los profesores de educación física que colaboraron en el estudio. Tras lo expuesto, la muestra final quedó conformada por un total de 1076 estudiantes, de los cuales un $58.64 \%$ eran mujeres y un $41.36 \%$ hombres. Los discentes se distribuían por niveles de la siguiente manera: un $31.51 \%$ de primero de la ESO; un $30.3 \%$ de segundo de la ESO; un $21.93 \%$ de tercero de la ESO; un $16.26 \%$ de cuarto de la ESO.

\section{Instrumento}

Para recabar los datos de este estudio se han utilizado dos instrumentos diferentes ya validados por otros estudios de la literatura científica. En primer lugar, se ha empleado el cuestionario FEFS-J (Engels \& Freund, 2019), el cual está compuesto por nueve ítems que se dividen en tres dimensiones (Placer; Experiencia de la actividad; Recreación). Las cuestiones presentadas en esta herramienta siguen la tipología Likert conformada por una escala de cero a tres, donde cero es «nunca» y tres es «siempre».

La validación de este cuestionario ya ha sido efectuada en varias investigaciones (Engels \& Freund, 2019; Lohbeck, Engels \& Freund, 2019). La fiabilidad y consistencia interna se ha realizado a través del alfa de Cronbach y el coeficiente de Omega, obteniendo así una escala global de consistencia interna y homogeneidad muy buena (á $=.85$; ù $=.89$ ). Por otro lado, si se atiende a cada una de las dimensiones del cuestionario, encontramos que las dimensiones de Placer y Recreación obtienen una consistencia interna buena (á =.86; ù=.91; $\mathrm{a}=.85 ; \mathrm{u}=.89)$; mientras que, solo aceptable para la dimensión Experiencia de la actividad (á $=65 ; \mathrm{ù}=.72)$.

La validación cuantitativa de este instrumento se hizo a través de un modelo g-CFA, el cual muestra la validez de los factores y las correlaciones entre estos. De este modo, este modelo mostró un ajuste aceptable del modelo, sin embargo, las correlaciones disminuyen (3-CFA: $\mathrm{rVE}+\mathrm{ER}=.88$; $\mathrm{rFL}+\mathrm{ER}$ 
$=.90 ; \mathrm{rVE}+\mathrm{FL}=.98)$, siento particularmente alta la establecida entre el placer y la experiencia de la actividad. Las cargas factoriales de este modelo de tres factores CFA se sitúan entre $\ddot{\mathrm{e}}=.54$ y .90 . En esta misma línea, se aplicaron otros modelos con correlaciones factoriales como el modelo threeESEM, el cual, en comparación con los resultados anteriores obtiene correlaciones factoriales ligeramente reducidas $(\mathrm{rVE}+\mathrm{ER}=.78 ; \mathrm{rFL}+\mathrm{ER}=.61 ; \mathrm{rVE}+\mathrm{FL}=.63)$. La varianza esclarecida se encuentra entre $\mathrm{R}^{2}=.29$ y .82 , por lo que solo la experiencia de la actividad (.46) se encuentra por debajo del valor .50. Si atendemos a la fiabilidad de construcción $(\mathrm{H})$, Hancock y Müller (2001) establecen que es de .72. Así, los factores placer $(\mathrm{H}=.91)$ y recuperación $(\mathrm{H}=.89)$ mostraron una alta fiabilidad de construcción, así como una varianza media declarada elevada $(\mathrm{DEVVE}=.76 \mathrm{y} \mathrm{DEVER}=.74)$.

Por otro lado, se ha empleado el cuestionario EDMCQPE (Milton, Appleton, Bryant \& Duda 2018), compuesto por 30 ítems que se dividen en cinco dimensiones (Tareas que implican; Autonomía y apoyo; Implicación; Control del profesor). Este cuestionario es de tipo Likert con una escala de cinco puntos, siendo uno «totalmente en desacuerdo» y cinco «totalmente de acuerdo».

La fiabilidad interna fue probada utilizando el alfa de Cronbach. Un alfa por encima de .80 constituye una medida fiable (Clark \& Watson, 1991), mientras que .70 y .60 en general son considerados como los límites más bajos para escalas con $>10$ artículos o $<10$ artículos, respectivamente (Cabello, Negro, Babin \& Anderson, 2010).

En este caso, también se valoró la opción de realizar la validación cuantitativa del instrumento a través de un modelo CFA o ESEM. Así, el análisis factorial de confirmación proporcionó un ajuste pobre, mientras que el ESEM un ajuste excelente a los datos de los cinco factores. El modelo ESEM también resultó en un factor más bajo $(|\mathrm{r}|=-.448 \mathrm{a}|\mathrm{r}|=$ .506) que el CFA $(|\mathrm{r}|=-.699 \mathrm{a}|\mathrm{r}|=$.939), lo que proporciona un mayor apoyo para el uso de ESEM con respecto al modelo CFA.

\section{Variables de estudio}

Aquí se recogen las distintas variables y dimensiones utilizadas en la investigación, junto con su abreviatura con la finalidad de facilitar la interpretación de los datos. Las variables (VAR) han sido: Valoración del uso de Aurasma en la clase de educación física (VAUR); Me gusta la clase de educación física (VE 01); La educación física me hace feliz (VE_02); La clase de educación física es buena para mí (VE_03); Las actividades en la clase de educación física me inspiran (FL_01); El tiempo vuela en la clase de educación física (FL 02); En la clase de educación física me estreso (FL_03); En la clase de educación física puedo recuperarme de otras asignaturas (ER_01); La educación física me ayuda a despejarme (ER 02); La educación física me da energía (ER_03); Mi profesor animó a probar nuevas habilidades (TE_01); Mi profesor intentó asegurarse de que los estudiantes se sintieran bien cuando daban lo mejor de sí mismos (TE_02); Mi profesor se aseguró que los estudiantes se sintieran exitosos cuando mejoraron (TE_03); Mi profesor reconoció a los estudiantes que se esforzaron (TE_04); Mi profesor se aseguró de que todos contribuyeran de manera importante (TE_05); Mi profesor se aseguró de que todos tuvieran un papel importante en el equipo (TE 06); Mi profesor se aseguró de que todos los jugadores son parte del éxito del equipo (TE_07); Mi profesor animó a ayudarse mutuamente a aprender (TE_08); Mi profesor animó a trabajar juntos como un equipo (TE_09); Mi profesor dio diversas opciones para desarrollar la actividad (AU_01); Mi profesor respondió completa y cuidadosamente a las preguntas de los estudiantes (AU 02); Cuando mi profesor pidió algo, intentó explicar por qué sería bueno hacerlo (AU_03); Mi profesor pensó que es importante practicar la actividad propuesta porque ellos disfrutan (AU_04); Se puede contar con mi profesor para lo que me preocupa, sin importar lo que haya ocurrido (AS_01); Mi profesor aprecia mucho a los estudiantes como personas y como alumnos (AS_02); Mi profesor escuchó y no juzgó los sentimientos personales de los alumnos (AS_03); Mi profesor cambió a los estudiantes cuando cometieron un error (IM_01); Mi profesor prestó más atención a los mejores estudiantes (IM_02); Mi profesor gritó a los estudiantes que se equivocaron (IM 03); Mi profesor tiene a sus estudiantes favoritos (IM_04); Mi profesor elogió a los estudiantes que tuvieron mejor rendimiento (IM_05); Mi profesor favorece a los mejores estudiantes (IM_06); Mi profesor es menos amistoso con aquellos que no hacían las cosas a su manera (CP_01); Mi profesor apoya menos a los que no hacen las cosas bien (CP_02); Mi profesor presta menos atención a los que no les cae bien (CP_03); Mi profesor acepta menos a los estudiantes que les decepcionan (CP_04); Mi profesor grita a los estudiantes delante de los demás para que hagan ciertas cosas (CP_05); Mi profesor castiga a los jugadores para mantenerlos en línea en las clase (CP_06); Mi profesor utiliza recompensas/alabanzas para que los estudiantes completen las tareas propuestas (CP_07); Mi profesor intenta interferir en la vida personal de los estudiantes (CP_08).

Las dimensiones utilizadas en el estudio han sido Placer (VE); Experiencia de la actividad (FL) y Recreación(ER) pertenecientes al cuestionario QUAEPE; y las dimensiones Tareas que implican (TE); Autonomía y Apoyo (AU); Apoyo social (AS), Implicación (IM) y control del profesor (CP) pertenecientes al cuestionario EDMCQ-PE.

\section{Procedimiento}

La primera fase se basó en secuenciar y temporalizar los contenidos colaborando con el departamento de Educación Física y Deportiva de los diferentes centros que participaron en esta investigación. Estos contenidos fueron extraídos de la programación didáctica para que se pudiera desarrollar diversas sesiones, un total de dos, durante el mes de septiembre del curso 2019-2020. El contenido trabajado está relacionado con la orientación espacial.

A continuación, se escogieron los espacios para desarrollar la orientación espacial y los puntos de orientación, buscando zonas de bastante extensión. La selección de puntos fue realizada por los docentes de la materia que se está trabajando, eligiendo un total de 15.

En una segunda fase se configuró el programa Aurasma. Lo primero que se realizó fue la creación de las imágenes activadoras; para ello, se tomaron fotografías de los puntos de orientación marcados en la fase anterior, generando así la imagen activadora, denominada trigger, para seguidamente 
geolocalizarla. Tras esta acción, se comenzaron a generar las capas virtuales de Realidad Aumentada, encargada durante todo este proceso de dar pistas a los alumnos para encontrar los distintos puntos de orientación.

Finalmente, se guardaron y compartieron el aura en un canal. Durante ese tramo de tiempo se generó el cuestionario mediante un formulario de Google Drive.

Se realizaron dos sesiones por cada grupo participante. En la primera sesión se explicó el programa Aurasma, su funcionamiento y su configuración en el teléfono móvil. También se crearon parejas de trabajo, utilizando como criterio el género y la disponibilidad de Smartphone de al menos uno de los miembros. En la segunda sesión se desarrolló la actividad en sí misma. Los grupos salían de dos en dos cada tres minutos y aquellos que terminaban la actividad antes que el resto servían de apoyo y guía para aquellos que presentaban más dificultad. Finalmente, se realizó un espacio de reflexión para valorar la actividad, además de facilitar el enlace de los cuestionarios. materia de educación física, ha sido media alta, considerando principalmente que el tiempo ha pasado muy rápido durante el desarrollo de la acción, y que no se han estresado durante el desarrollo de la actividad (tabla 1).

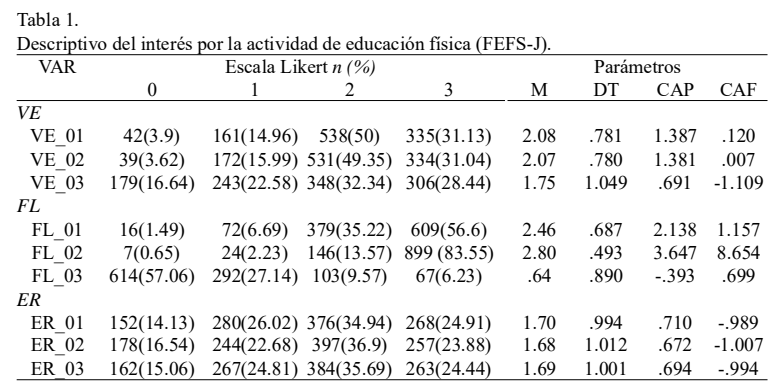

Con respecto a la motivación presentada por los alumnos, esta se sitúa en valores medio-alto en todas las dimensiones, excepto en la dimensión de control del profesorado, donde las puntuaciones son bajas, tal y como se observa en la tabla 2 .

\section{Análisis de datos}

Se ha utilizado el programa estadístico StatisticalPackageforthe Social Sciences (SPSS) versión 24. Esto permitió la realización de un análisis en profundidad de las distintas variables expuestas. En concreto, para el estudio descriptivo se han empleado estadísticos como el recuento de frecuencias, la media (M), desviación típica (DT) y pruebas específicas para hallar la tendencia de la distribución como el coeficiente de asimetría de Pearson (CAP) y de apuntamiento de Fisher (CAF).

La comparación de variables del análisis correlacional se ha realizado mediante el test Chi-cuadrado de Pearson $\left(\div^{2}\right)$. Para determinar la fuerza de asociación se ha utilizado la $\mathrm{V}$ de Cramer (V) y el coeficiente de contingencia (Cont). Asimismo, se ha llevado a cabo un modelo de regresión lineal múltiple para predecir el efecto de las variables independientes VAUR sobre las variables dependiente FEFS-J y EDMCQ-PE. Las diferencias estadísticamente significativas se han definido a partir de $p<.05$.

\section{Resultados}

Los resultados que se presentan a continuación se obtienen de los diversos análisis estadísticos aplicados. En la figura 1 se observa como la mayoría de los estudiantes valoran positivamente la actividad $(78.53 \%)$, mientras que una minoría lo hace de forma negativa $(21.47 \%)$

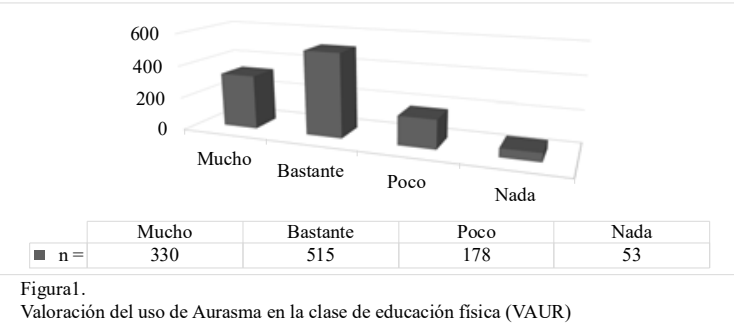

Las valoraciones dadas por los estudiantes, en relación al interés por el desarrollo de la actividad con Aurasma en la

\begin{tabular}{|c|c|c|c|c|c|c|c|c|c|}
\hline \multirow{2}{*}{ VAR } & \multicolumn{5}{|c|}{ scala Likert $n$} & \multicolumn{4}{|c|}{ Parámetros } \\
\hline & 1 & 2 & 3 & 4 & 5 & $\mathrm{M}$ & DT & CAP & $\mathrm{CAF}$ \\
\hline \multicolumn{10}{|l|}{$\overline{T E}$} \\
\hline TE 01 & $101(9.39)$ & $125(11.62)$ & 199(18.49) & $293(27.23)$ & $358(33.27)$ & 3.63 & 1.301 & 2.024 & -0.718 \\
\hline TE 02 & $139(12.92)$ & $143(13.29)$ & $179(16.64)$ & $268(24.91)$ & $347(32.25)$ & 3.50 & 1.391 & 1.798 & -1.027 \\
\hline TE-03 & $166(15.43)$ & $158(14.68)$ & $168(15.61)$ & $245(22.77)$ & $339(31.51)$ & 3.40 & 1.445 & 1.661 & -1.215 \\
\hline TE_04 & $74(6.88)$ & $116(10.78)$ & $232(21.56)$ & $298(27.7)$ & $356(33.09)$ & 3.69 & 1.225 & 2.197 & -0.554 \\
\hline TE_-05 & $15(1.39)$ & $53(4.93)$ & $168(15.61)$ & $334(31.04)$ & $506(47.03)$ & 4.17 & 0.958 & 3.311 & 0.590 \\
\hline TE 06 & $23(2.14)$ & $\begin{array}{l}61(5.67) \\
6\end{array}$ & $172(15.99)$ & $324(30.11)$ & $496(46.1)$ & 4.12 & 1.012 & 3.085 & 0.537 \\
\hline TE 07 & $153(14.22)$ & $179(16.64)$ & $166(15.43)$ & $224(20.82)$ & $354(32.9)$ & 3.41 & 1.444 & 1.672 & -1.252 \\
\hline TE 08 & $19(1.77)$ & $47(4.37)$ & $93(8.64)$ & $176(16.36)$ & 741(68.87) & 4.46 & 0.946 & 3.658 & 2.738 \\
\hline TE 09 & $11(1.02)$ & $36(3.35)$ & $84(7.8$ & $192(1$ & $753(69.98)$ & 4.52 & 0.853 & 4.127 & 3.409 \\
\hline \multicolumn{10}{|c|}{ 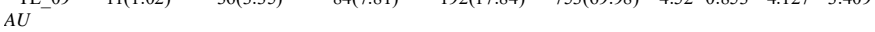 } \\
\hline AU_01 & $182(16.91)$ & $171(15.89)$ & 204(18.96) & $146(13.57)$ & $373(34.67)$ & 3.33 & 1.499 & 1.555 & -1.374 \\
\hline $\mathrm{AU}_{-}^{-02}$ & $7(0.65)$ & $21(1.95)$ & $62(5.76)$ & $290(26.95)$ & $696(64.68)$ & 4.53 & 0.747 & 4.720 & 3.966 \\
\hline $\mathrm{AU}_{-}^{-} 03$ & $11(1.02)$ & $32(2.97)$ & 59 & $318(29.55)$ & $656(60.97)$ & 4.46 & 0.812 & 4.266 & 3.636 \\
\hline AU_04 & $92(8.55)$ & $117(10.87)$ & 187(17.38) & $291(27.04)$ & $389(36.15)$ & 3.71 & 1.287 & 2.107 & -0.594 \\
\hline \multicolumn{10}{|l|}{$A S$} \\
\hline AS 01 & $149(13.85)$ & $163(15.15)$ & $228(21.19)$ & 196(18.22) & $340(31.6)$ & 3.38 & 1.415 & 1.685 & -1.199 \\
\hline AS 02 & $175(16.26)$ & $183(17$. & $209(19$ & $218(2$ & $291(2$ & 3.24 & 1.429 & 1.572 & -1.280 \\
\hline $\mathrm{AS}_{-} 03$ & $231(21.47)$ & $206(19.14)$ & $212(19.7)$ & $254(23.61)$ & $173(16.08)$ & 2.93 & 1.387 & 1.395 & -1.278 \\
\hline \multicolumn{10}{|c|}{ 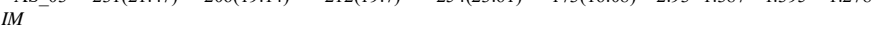 } \\
\hline IM_01 & $284(26.39)$ & $276(26)$ & $194(18$. & 169 & 153( & 2.65 & 1.385 & 1.195 & -1.146 \\
\hline $\mathrm{IM}_{-}^{-} 02$ & $279(25.93)$ & $293(27.23)$ & $204(18.96)$ & $153(14.22)$ & $147(13.66)$ & 2.62 & 1.362 & 1.192 & -1.054 \\
\hline IM_-03 & $394(36.62)$ & $324(30.11)$ & $249(23.14)$ & $61(5.67)$ & $48(4.46)$ & 2.11 & 1.101 & 1.009 & 0.074 \\
\hline IM_04 & $206(19.14)$ & $284(26.39)$ & $271(25.19)$ & $196(18.22)$ & $119(11.06)$ & 2.75 & 1.262 & 1.390 & -0.976 \\
\hline IM_05 & $16(1.49)$ & $53(4.93$ & $99(9.2)$ & $481(44.7)$ & $427(39.68)$ & 4.16 & 0.890 & 3.549 & 1.645 \\
\hline IM_06 & $319(29.65)$ & $328(30.48)$ & $220(20.45)$ & $108(10.04)$ & $101(9.39)$ & 2.39 & 1.262 & 1.100 & -0.574 \\
\hline \multicolumn{10}{|l|}{$C P$} \\
\hline CP_ 01 & $371(34.48)$ & $344(31.97)$ & $284(26.39)$ & $61(5.67)$ & $16(1.49)$ & 2.07 & 0.981 & 1.097 & -0.289 \\
\hline $\mathrm{CP}_{-} 02$ & $382(35.5)$ & $359(33.36)$ & $218(20$. & $82(7.62)$ & $35(3$ & 2.09 & 1.070 & 1.025 & 0.014 \\
\hline $\mathrm{CP}_{-} 03$ & $418(38.85)$ & $378(35.13)$ & $153(14.22)$ & $91(8.46)$ & $36(3.35)$ & 2.02 & 1.081 & 0.946 & 0.270 \\
\hline $\mathrm{CP}_{-}^{-} 04$ & $420(39.03)$ & $361(33.55)$ & $168(15.61)$ & $74(6.88)$ & $53(4.93)$ & 2.05 & 1.123 & 0.935 & 0.338 \\
\hline $\mathrm{CP}_{-}^{-} 05$ & $435(40.43)$ & $365(33.92)$ & $163(15$. & $62(5.76$ & $51(4.74)$ & 2.01 & 1.101 & 0.912 & 0.596 \\
\hline $\mathrm{CP} 06$ & $401(37.27)$ & $399(37.08)$ & $172(15.99)$ & $54(5.02)$ & $50(4.65)$ & 2.02 & 1.072 & 1.150 & 1.072 \\
\hline CP_07 & $162(15.06)$ & $183(17.01)$ & $280(26.02)$ & $263(24.44)$ & $188(17.47)$ & 3.12 & 1.304 & 1.627 & -1.049 \\
\hline $\mathrm{CP} 08$ & $583(54.18)$ & $405(37.64)$ & $\begin{array}{l}54(5.02) \\
\end{array}$ & $28(2.6)$ & $6(0.56)$ & 1.57 & 0.753 & 0.766 & 3.024 \\
\hline
\end{tabular}

Se observa relación de significancia entre las variables que conforman FEFS-J con la variable VAUR, aunque la fuerza de relación es baja, según marcan los valores dados por el estadístico V de Cramer (tabla 3).

En las correlaciones establecidas entre la valoración del programa Aurasma y las tareas que implican su desarrollo, se muestra relación de significancia en todos los ítems, excepto en el hecho de reconocer que los estudiantes se esforzaron durante su desarrollo. En las variables donde hay correlación, la fuerza de relación es baja $(<.02)$, excepto en la variable en la que el profesor asegura que todos los estudiantes forman parte del equipo, cuya relación es media $(<.06)$ según el estadístico V de Cramer (tabla 4).

Las correlaciones establecidas entre las variables que conforman la dimensión autonomía y apoyo, apoyo social e implicación con la variable de valoración del uso de Aurasma, 


\begin{tabular}{|c|c|c|c|c|c|c|c|c|}
\hline \\
\hline \multirow[t]{2}{*}{ VAR } & \multicolumn{4}{|c|}{ Escala Likert $n(\%)$} & \multicolumn{4}{|c|}{ Parámetros } \\
\hline & 0 & 1 & ( & 3 & ?2(gl) & $p$-va. & Con & $\mathrm{V}$ \\
\hline VE 01 & & & & & $75.79(9)$ & $<.000$ & .257 & .153 \\
\hline MŪC & $7(0.65)$ & $54(5.02)$ & $155(14.41)$ & $144(13.38)$ & & & & \\
\hline BAS & $9(0.84)$ & $67(6.23)$ & $287(26.67)$ & $152(14.13)$ & & & & \\
\hline POC & $15(1.39)$ & $27(2.51)$ & $84(7.81)$ & $52(4.83)$ & & & & \\
\hline NAD & $11(1.02)$ & $13(1.21)$ & $12(1.12)$ & $17(1.58)$ & & & & \\
\hline VE 02 & & & & & $96.60(9)$ & $<.000$ & .287 & .173 \\
\hline MUC & $6(0.56)$ & $55(5.11)$ & $153(14.22)$ & $116(10.78)$ & & & & \\
\hline BAS & $6(0.56)$ & $74(6.88)$ & $285(26.49)$ & $150(13.94)$ & & & & \\
\hline POC & $16(1.49)$ & $25(2.32)$ & $80(7.43)$ & $57(5.3)$ & & & & \\
\hline NAD & $11(1.02)$ & $18(1.67)$ & $13(1.21)$ & $11(1.02)$ & & & & \\
\hline VE_03 & & & & & $33.95(9)$ & $<.000$ & .175 & .103 \\
\hline MŪC & $45(4.18)$ & $73(6.78)$ & $111(10.32)$ & $101(9.39)$ & & & & \\
\hline BAS & $87(8.09)$ & $128(11.9)$ & $157(14.59)$ & $143(13.29)$ & & & & \\
\hline POC & $25(2.32)$ & $31(2.88)$ & $68(6.32)$ & $54(5.02)$ & & & & \\
\hline NAD & $22(2.04)$ & $11(1.02)$ & $12(1.12)$ & $8(0.74)$ & & & & \\
\hline FL_ 01 & & & & & $35.47(9)$ & $<.000$ & .179 & .105 \\
\hline MUC & $4(0.37)$ & $21(1.95)$ & $118(10.97)$ & $187(17.38)$ & & & & \\
\hline BAS & $5(0.46)$ & $28(2.6)$ & $171(15.89)$ & $311(28.9)$ & & & & \\
\hline $\mathrm{POC}$ & $3(0.28)$ & $13(1.21)$ & $75(6.97)$ & $87(8.09)$ & & & & \\
\hline NAD & $4(0.37)$ & $10(0.93)$ & $15(1.39)$ & $24(2.23)$ & & & & \\
\hline FL_02 & & & & & $50.98(9)$ & $<.000$ & .213 & .126 \\
\hline MUEC & $0(0)$ & $5(0.46)$ & $39(3.62)$ & $286(26.58)$ & & & & \\
\hline BAS & $4(0.37)$ & $9(0.84)$ & $49(4.55)$ & $453(42.1)$ & & & & \\
\hline POC & $2(0.19)$ & $6(0.56)$ & $43(4)$ & $127(11.8)$ & & & & \\
\hline NAD & $1(0.09)$ & $4(0.37)$ & $15(1.39)$ & $33(3.07)$ & & & & \\
\hline FL 03 & & & & & $54.26(9)$ & $<.000$ & .219 & .130 \\
\hline MUC & $219(20.35)$ & $66(6.1$ & $24(2$. & $21(1.95)$ & & & & \\
\hline BAS & $302(28.07)$ & $149(13.85)$ & $41(3.81)$ & $23(2.14)$ & & & & \\
\hline POC & $77(7.16)$ & $59(5.48)$ & $26(2.42)$ & $16(1.49)$ & & & & \\
\hline NAD & $16(1.49)$ & $18(1.67)$ & $12(1.12)$ & $7(0.65)$ & & & & \\
\hline ER_01 & & & & & $55.25(9)$ & $<.000$ & .221 & .131 \\
\hline MŪC & $49(4.55)$ & $71(6$. & $115(10.69)$ & $95(8.8$ & & & & \\
\hline BAS & $46(4.28)$ & $147(13.66)$ & $208(19.33)$ & $114(10$ & & & & \\
\hline POC & $47(4.37)$ & $49(4.55)$ & $34(3.16)$ & $48(4.46)$ & & & & \\
\hline NAD & $10(0.93)$ & $13(1.21)$ & $19(1.77)$ & $11(1.02)$ & & & & \\
\hline ER_02 & & & & & $64.54(9)$ & $<.000$ & .238 & .141 \\
\hline MŪC & $42(3.9)$ & $79(7.34)$ & $118(10.97)$ & $91(8.46)$ & & & & \\
\hline BAS & $63(5.86)$ & $121(11.25)$ & $217(20.17)$ & $114(1 \mathrm{C}$ & & & & \\
\hline POC & $61(5.67)$ & $35(3.25)$ & $39(3.62)$ & $43(4)$ & & & & \\
\hline NAD & $12(1.12)$ & $9(0.84)$ & $23(2.14)$ & $9(0.84)$ & & & & \\
\hline ER_03 & & & & & $27.95(9)$ & .001 & .159 & .093 \\
\hline MUC & $49(4.55)$ & $84(7.81)$ & $141(13.1)$ & $56(5.2)$ & & & & \\
\hline BAS & $68(6.32)$ & $123(11.43)$ & $176(16.36)$ & $148(13.75)$ & & & & \\
\hline POC & $34(3.16)$ & $56(5.2)$ & $57(5.3)$ & $41(3$. & & & & \\
\hline NAD & $11(1.02)$ & $14(1.3)$ & $10(0.93)$ & $18(1.67)$ & & & & \\
\hline
\end{tabular}

tal y como se muestra en la tabla 5, mantienen relación de significancia, aunque la fuerza de asociación es baja $(<.02)$, excepto en las diversas opciones dadas por el docente para desarrollar la actividad, el cambio de parejas, la atención

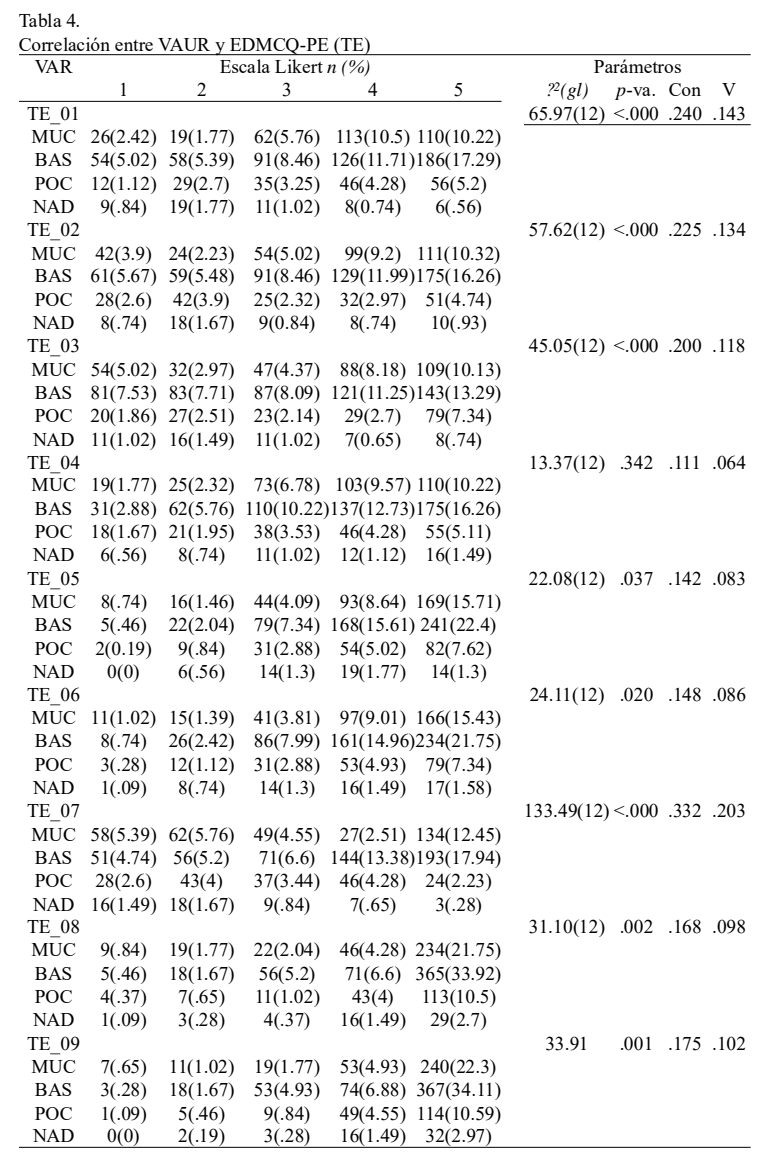

mostrada por el docente a los mejores estudiantes y el favoritismo por parte del profesor a los mejores estudiantes, donde la relación es media $(<.06)$, teniendo en cuenta lo establecido por la prueba $\mathrm{V}$ de Cramer.

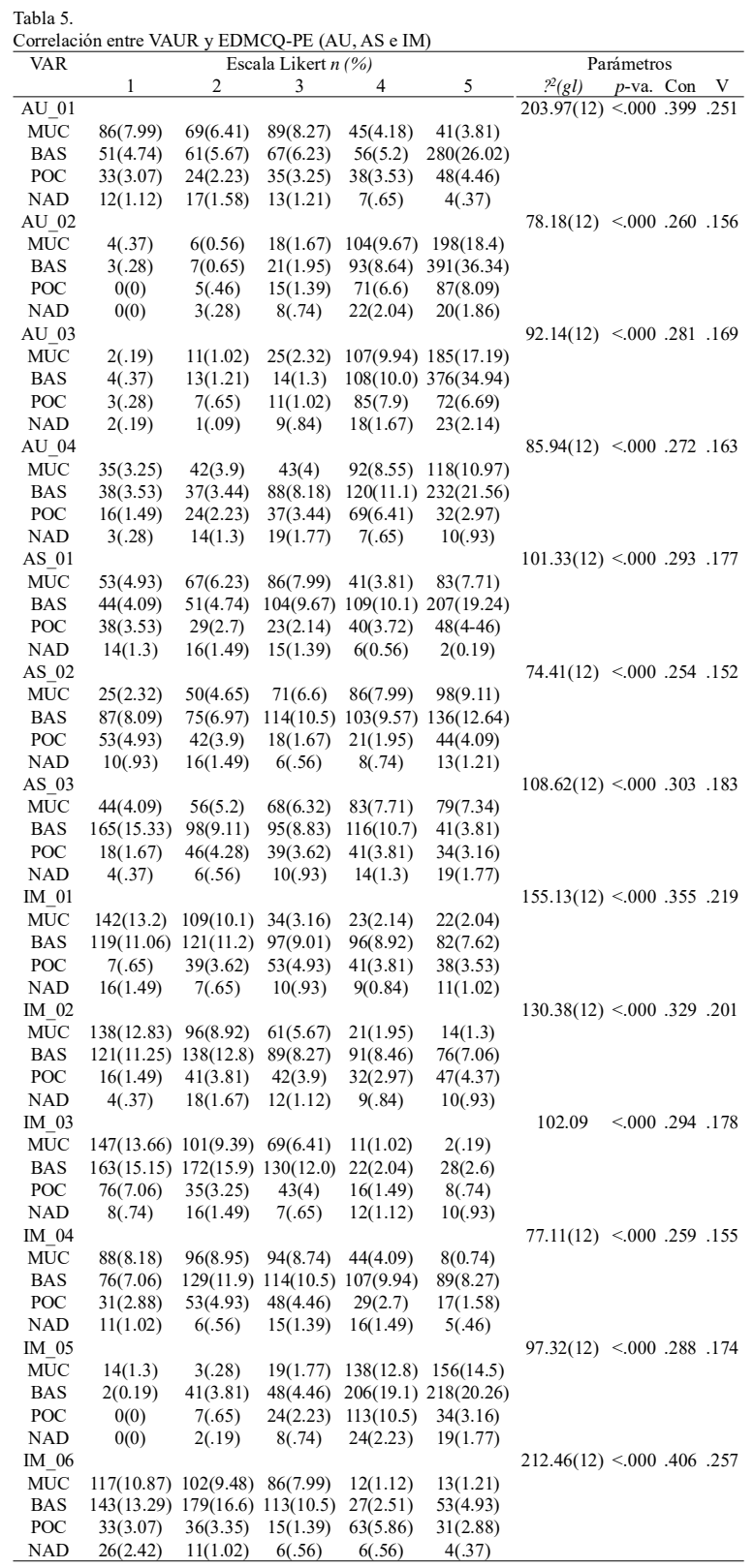

En la relación marcada entre la valoración del uso de Aurasma y las variables que conforman la dimensión de control por parte del profesor, las relaciones establecidas muestran significancia estadística, aunque la fuerza de conexión es baja en todas ellas $(<.02)$ excepto en la variable que marca que el docente trata de interferir en la vida personal de los estudiantes, cuya fuerza de asociación es media $(<.02)$, según el estadístico $\mathrm{V}$ de Cramer (tabla 6).

En relación al efecto de las variables independientes que conforma el cuestionario FEFS-J y la valoración del uso de Aurasma, los estadísticos empleados en el modelo de regresión múltiple alcanzan la significancia $[F(153.751)=32.273$; $p<.001$ ], revelando el $21.4 \%$ de la varianza. Entre las variables que reflejan un efecto significativo y que condicionan a la variable dependiente VAUR, la que muestra una mayor 


\begin{tabular}{|c|c|c|c|c|c|c|c|c|}
\hline \multirow{2}{*}{\multicolumn{6}{|c|}{ Likert n (\%) }} & \multirow{2}{*}{\multicolumn{3}{|c|}{ Parámetros }} \\
\hline & & & & & & & & \\
\hline & \multicolumn{5}{|c|}{$\begin{array}{ll}\text { Likert n }(\%) \\
3\end{array}$} & \multicolumn{3}{|c|}{$? 2(\mathrm{gl}) \quad p$-va. Con $\mathrm{V}$} \\
\hline CP_01 & & & & & & $95.70(12)<.000$ & .286 & .172 \\
\hline MÜC & $126(11.71)$ & $114(10.59)$ & $76(7.06)$ & $13(1.21)$ & $1(.09)$ & & & \\
\hline BAS & $181(16.82)$ & $160(14.87)$ & $152(14.13)$ & $19(1.77)$ & $3(.28)$ & & & \\
\hline POC & $46(4.28)$ & $59(5.48)$ & $47(4.37)$ & $21(1.95)$ & $5(.46)$ & & & \\
\hline NAD & $18(1.67)$ & $11(1.02)$ & $9(.84)$ & $8(.74)$ & $7(.65)$ & & & \\
\hline CP_02 & & & & & & $62.49(12)<.000$ & .234 & .139 \\
\hline MŪC & $123(11.43)$ & $127(11.8)$ & $47(4.37)$ & $28(2.6)$ & $5(.46)$ & & & \\
\hline BAS & $188(17.47)$ & $169(15.71)$ & $121(11.25)$ & $22(2.04)$ & $15(1.39)$ & & & \\
\hline $\mathrm{POC}$ & $54(5.02)$ & $51(4.74)$ & $44(4.09)$ & $19(1.77)$ & $10(.93)$ & & & \\
\hline NAD & $17(1.58)$ & $12(1.12)$ & $6(.56)$ & $13(1.21)$ & $5(.46)$ & & & \\
\hline CP_03 & & & & & & $90.91(12)<.000$ & .279 & .168 \\
\hline MŪC & $112(10.41)$ & $152(14.13)$ & $33(3.07)$ & $29(2.7)$ & $4(.37)$ & & & \\
\hline BAS & $224(20.82)$ & $171(15.89)$ & $81(7.53)$ & $26(2.42)$ & $13(1.21)$ & & & \\
\hline POC & $63(5.86)$ & $46(4.28)$ & $32(2.97)$ & $21(1.95)$ & $16(1.49)$ & & & \\
\hline NAD & $19(1.77)$ & $9(0.84)$ & $7(.65)$ & $15(1.39)$ & $3(.28)$ & & & \\
\hline CP_04 & & & & & & $83.09(12)<.000$ & .268 & .160 \\
\hline MŪC & $123(11.43)$ & 143(13.29) & $20(1.86)$ & $28(2.6)$ & $16(1.49)$ & & & \\
\hline BAS & $218(20.26)$ & $168(15.61)$ & $89(8.27)$ & $23(2.14)$ & $17(1.58)$ & & & \\
\hline POC & $62(5.76)$ & $35(3.25)$ & $51(4.74)$ & $16(1.49)$ & $14(1.3)$ & & & \\
\hline NAD & $17(1.58)$ & $15(1.39)$ & $8(.74)$ & $7(.65)$ & $6(.56)$ & & & \\
\hline CP_05 & & & & & & $71.12(12)<.000$ & .249 & .148 \\
\hline MŪC & $127(11.8)$ & $145(13.48)$ & $19(1.77)$ & $24(2.23)$ & $15(1.39)$ & & & \\
\hline BAS & $221(20.54)$ & $167(15.52)$ & $87(8.09)$ & $22(2.04)$ & $18(1.67)$ & & & \\
\hline POC & $67(6.23)$ & $39(3.62)$ & $49(4.55)$ & $10(.93)$ & $13(1.21)$ & & & \\
\hline NAD & $20(1.86)$ & $14(1.3)$ & $8(.74)$ & $6(.56)$ & $5(.46)$ & & & \\
\hline CP_06 & & & & & & $57.81(12)<.000$ & .226 & .134 \\
\hline MŨC & $112(10.41)$ & $159(14.78)$ & $23(2.14)$ & $19(1.77)$ & $17(1.58)$ & & & \\
\hline BAS & $208(19.33)$ & $173(16.08)$ & $96(8.92)$ & $17(1.58)$ & $21(1.95)$ & & & \\
\hline POC & 63(5.86) & $52(4.83)$ & $43(4)$ & $11(1.02)$ & $9(.84)$ & & & \\
\hline NAD & $18(1.67)$ & $15(1.39)$ & $10(.93)$ & $7(.65)$ & $3(.28)$ & & & \\
\hline CP 07 & & & & & & $41.59(12)<.000$ & .193 & .114 \\
\hline MÜC & $41(3.81)$ & $45(4.18)$ & $86(7.99)$ & $74(6.88)$ & $84(7.81)$ & & & \\
\hline BAS & $72(6.69)$ & $89(8.27)$ & $143(13.29) 1$ & $136(12.64)$ & $75(6.97)$ & & & \\
\hline POC & $36(3.35)$ & $37(3.44)$ & $42(3.9)$ & $46(4.28)$ & $17(1.58)$ & & & \\
\hline NAD & $13(1.21)$ & $12(1.12)$ & $9(.84)$ & $7(.65)$ & $12(1.12)$ & & & \\
\hline CP 08 & & & & & & $159.38(12)<.000$ & .359 & .222 \\
\hline MŨC & $141(13.1)$ & $168(15.61)$ & $9(0.84)$ & $8(0.74)$ & $4(.37)$ & & & \\
\hline BAS & $296(27.51)$ & $186(17.29)$ & $15(1.39)$ & $16(1.49)$ & $2(.19)$ & & & \\
\hline $\mathrm{POC}$ & $134(12.45)$ & $26(2.42)$ & $14(1.3)$ & $4(.37)$ & $0(0)$ & & & \\
\hline NAD & $12(1.12)$ & $25(2.32)$ & $16(1.49)$ & $0(0)$ & $0(0)$ & & & \\
\hline
\end{tabular}

Tabla 7

Efecto de las variables independientes FEFS-J sobre VAUR

\begin{tabular}{lrrr}
\hline Variables & $B(E T)$ & $t$ & $p$-valor \\
\hline VE & $.237(.144)$ & 2.263 & .024 \\
VE_01 & $-.829(.140)$ & -5.904 & .000 \\
VE_02 & $.077(.068)$ & 1.136 & .256 \\
VE_03 & & & \\
FL & $.124(.69)$ & 1.792 & .073 \\
FL_01 & $.147(.080)$ & 1.831 & .067 \\
FL_02 & $.503(.040)$ & 12.489 & .000 \\
FL_03 & & & \\
ER & $.211(.105)$ & 2.017 & .044 \\
ER_01 & $-.667(.098)$ & -6.788 & .000 \\
ER_02 & $.229(.052)$ & 4.366 & .000 \\
ER_03 & & & \\
\hline
\end{tabular}

\begin{tabular}{|c|c|c|c|}
\hline Variables & $B(E T)$ & $t$ & $p$-valor \\
\hline \multicolumn{4}{|l|}{ TE } \\
\hline TE_01 & $-.521(.045)$ & -11.676 & .000 \\
\hline TE 02 & $.095(.047)$ & 2.008 & .045 \\
\hline TE 03 & $.087(.042)$ & 2.091 & .037 \\
\hline TE_04 & $-.071(.052)$ & -1.352 & .177 \\
\hline TE_-05 & $.177(.058)$ & 3.030 & .003 \\
\hline TE_06 & $-.071(.061)$ & -1.167 & .243 \\
\hline TE_07 & $-.166(.039)$ & -4.288 & .000 \\
\hline TE_08 & $.219(.059)$ & 3.722 & .000 \\
\hline TE_09 & $.192(.062)$ & 3.097 & .002 \\
\hline \multicolumn{4}{|l|}{$\mathrm{AU}$} \\
\hline AU 01 & $.075(.035)$ & 2.150 & .032 \\
\hline AU 02 & $-.056(.057)$ & -.996 & .320 \\
\hline AU_03 & $-.222(.052)$ & -4.260 & .000 \\
\hline AU_04 & $.145(.043)$ & 3.399 & .001 \\
\hline \multicolumn{4}{|l|}{ AS } \\
\hline AS_01 & $.007(.042)$ & .179 & .858 \\
\hline AS_02 & $-.463(.036)$ & -12.693 & .000 \\
\hline AS 03 & $.080(.035)$ & 2.271 & .023 \\
\hline \multicolumn{4}{|l|}{ IM } \\
\hline IM_01 & $-.014(.043)$ & -.337 & .736 \\
\hline IM_02 & $.451(.044)$ & 10.158 & .000 \\
\hline IM_03 & $.439(.042)$ & 10.421 & .000 \\
\hline IM_04 & $.204(.037)$ & 5.591 & .000 \\
\hline IM_05 & $.258(.043)$ & 5.993 & .000 \\
\hline IM_06 & $-.074(.034)$ & -2.210 & .027 \\
\hline \multicolumn{4}{|l|}{$\mathrm{CP}$} \\
\hline CP 01 & $.413(.051)$ & 8.156 & .000 \\
\hline $\mathrm{CP} 02$ & $-.167(.056)$ & -2.964 & .003 \\
\hline $\mathrm{CP} 03$ & $.342(.051)$ & 6.750 & .000 \\
\hline $\mathrm{CP}_{-}^{-} 04$ & $.032(.067)$ & .476 & .634 \\
\hline CP_05 & $-.314(.067)$ & -4.688 & .000 \\
\hline CP_06 & $-.144(.057)$ & -2.514 & .012 \\
\hline $\mathrm{CP} 07$ & $-.407(.041)$ & -9.891 & .000 \\
\hline $\mathrm{CP} 08$ & $-.335(.039)$ & -8.687 & .000 \\
\hline
\end{tabular}

influencia es la variable independiente la educación física me da energía para otras cosas (tabla 7).

Con respecto a la relación entre las variables que conforman el cuestionario EDMCQ-PE sobre la variable VAUR (Tabla 8), los estadísticos empleados en el modelo de regresión múltiple alcanzan la significancia $[F(565.982)=129.66 ; p<$ $.001]$, revelando el $78.8 \%$ de la varianza. Entre las variables que reflejan un efecto significativo y que condicionan a la variable dependiente VAUR, las que mayores valores presentan son mi profesor prestó más atención a los mejores estudiantes, mi profesor gritó a los estudiantes que se equivocaron y mi profesor es menos amistoso con los estudiantes que no hacían las cosas a su manera.

\section{Discusión y conclusiones}

La inclusión de la tecnología en los procesos de enseñanza y aprendizaje es una realidad hoy en día en los centros educativos, debido a que promueven prácticas educativas innovadoras, provocando el aumento de la participación, interés y motivación de los propios estudiantes, tal y como ya indicaron Area, Hernández \& Sosa (2016), Cabero \& Barroso (2018), Castañeda, Esteve \& Adell (2018), Fombona \& Pascual (2017), Fuentes, lópez \& Pozo (2019), Marín \& Muñoz (2018), Radu (2014), Rodríguez, Cáceres \& Alonso (2018), Villalustres \& Del Moral (2017), Viñals \& Cuenta (2016).

Uno de esos recursos tecnológicos es la realidad aumentada, la cual potencia la experimentación, la competencia digital y el trabajo colaborativo, generando con ello un aprendizaje significativo, constructivista y por descubrimiento, teniendo presente lo establecido por Akcayir \& Akcayir (2017), Barroso, Cabero, García, Calle, Gallego \& Casado (2017), Cabero \& Roig (2019), Chen, Liu, Cheng \& Huang (2017), Garay, Tejada \& Castaño (2017), Gómez, Trujillo,Aznar \& Cáceres (2018), López, Pozo \& López (2019), Rodríguez Hinojo \& Agreda (2019), no pasando desapercibido por las distintas etapas y ramas educativas (Álvarez, Castillo \& Geldes, 2017; Heradio, De la Torre, Galán, Cabrerizo, Herrera \& Dormido, 2016; Jaramillo, Silva, Velásquez, Páramo \& Gómez, 2018; Lorenzo \& Scagliarini, 2018; Muhamedyev, Aliguliyev, Shokishalov \& Mustakayev, 2018; Suh \& Propeht, 2018)

Todo esto se ve reflejado en el presente estudio ya que el alumnado participante ha valorado positivamente el uso de Aurasma en la sesión dedicada a la orientación espacial, estando en consonancia a lo marcado por Roig-Vila, Lorenzo-Lledó \& Mengual-Andrés (2019).

El interés mostrado por los discentes en el desarrollo de la sesión ha sido positivo, siendo su principal sensación que el tiempo ha pasado muy rápido para ellos y que la actividad le ha inspirado, siguiendo en la línea marcada por Delello (2014). Y, además, sin padecer estrés.

Su motivación de cara al desarrollo de la tarea también ha sido óptima, dado que las valoraciones dadas por los estudiantes se sitúan en un umbral medio-alto, sobre todo en la implicación hacia la tarea y la autonomía en su desarrollo. Estos han destacado el elogio de los docentes hacia ellos, el ayudarse en la tarea, trabajar en equipo, la explicación del docente y su valoración positiva de las acciones a desarrollar; presentando además una relación baja con respecto al 
control que tiene el docente hacia ellos, coincidiendo con Ruiz-Ariza, Casuso, Suárez-Manzano \& Martínez-López (2018).

Dado los resultados obtenidos, se puede indicar que existe relación entre la valoración dada por los estudiantes en relación al uso de Aurasma durante la sesión, con el interés mostrado hacia la tarea y su motivación, habiendo una influencia directa y consistente sobre la energía que le da la materia de Educación Física para el desarrollo de otras tareas. Además, los estudiantes han valorado positivamente la actitud de los docentes hacia ellos, estando relacionado con lo marcado por Muñoz-Cristobal, Gallego-Lema, Arribas-Cubero, Martínez-Mones \& Asensio-Pérez (2017).

Se concluye que el uso de Aurasma en las sesiones de Educación Física para el desarrollo de la orientación espacial es valorado positivamente por los estudiantes en la etapa de la ESO, mostrando interés y motivación por la asignatura. Además, la motivación se ve mucho más influenciada por el uso de Aurasma, que el interés por la materia.

Finalmente, es importante señalar las limitaciones que se han encontrado a la hora de realizar esta investigación. Así, se destaca la dificultad a la hora de elaborar, diseñar y organizar los materiales creados para el desarrollo de la actividad propuesta en el estudio. Del mismo modo, se ha tenido que dotar al profesorado participante de una formación específica en el uso de la tecnología concreta que se ha implementado y estudiado. Además, el grupo de discentes que componen la muestra es un grupo ya preestablecido con una dificultad añadida, el momento en el que se realiza la actividad ya que es con el curso comenzado, por lo que es imposible la modificación del grupo y la realización de un muestreo aleatorio.

La prospectiva de la investigación es ofrecer un método de enseñanza activo para aquellos docentes que quieran desarrollar y enseñar el contenido de la orientación espacial a su alumnado. Se han encontrado limitaciones al realizar la recopilación de la muestra, explicar del método de enseñanza a los docentes y buscar soluciones para la falta de recursos tecnológicos necesarios para el desarrollo de la actividad.

Como futura línea de investigación se propone estudiar la incidencia de la RA para el desarrollo de otros contenidos.

\section{Referencias}

Akcayir, M., \& Akcayir, G. (2017). Advantages and challenges associated with augmented reality crossMark for education: A systematic review of the literature. Educational Research Review, 20,1-11.doi: 10.1016/j.edurev.2016.11.002

Álvarez,A., Castillo,M., \& Geldes, C. (2017).Análisis Bibliométrico de la Realidad Aumentada y su Relación con la Administración de Negocios. Información tecnológica, 28(4), 57-66. http://dx.doi.org/10.4067/S0718-07642017000400008

Area, M., Hernández, V., \& Sosa, J. J. (2016). Modelos de integración didáctica de las TIC en el aula. Comunicar: Revista científica iberoamericana de comunicación y educación, 24(47), 79-87.http://dx.doi.org/10.3916/C47-2016-08

Aznar, I., Cáceres, M. P., Trujillo, J. M, \& Romero, J. M. (2019). Impacto de las apps móviles en la actividad física: un metaanálisis. Retos: nuevas tendencias en educación física, deporte yrecreación, (36), 52-57.

Barroso, J., Cabero, J., García, F., Calle, F. M., Gallego, O., \& Casa- do, I. (2017). Diseño, producción, evaluación y utilización educativa de la realidad aumentada. Sevilla: Secretariado de Recursos Audiovisuales y NNTT. Universidad de Sevilla.

Cabero, J., \& Barroso, J. (2018). Los escenarios tecnológicos en Realidad Aumentada (RA): posibilidades educativas en estudios universitarios. Aula Abierta, 47(3), 327-336.

Cabero, J., \& Infante,A. (2014). Empleo del método Delphi y su empleo en la investigación en comunicación y educación. Edutec, 48, 1-16. doi: 10.21556/edutec.2014.48.187

Cabero, J., Llorente, C., \& Gutiérrez, J. J. (2017). Evaluación pory desde los usuarios: objetos de aprendizaje con Realidad aumentada. Revista de Educación a Distancia, (53), 1-17.

Cabero, J., Llorente, M. C., \& Marín, V. (2017). Comunidades virtuales de aprendizaje. El Caso del proyecto de realidad aumentada: RAFODIUM. Perspectiva Educacional. Formación de Profesores, 56(2), 117-138.

Cabero, J., \& Roig, R. (2019). The motivation of technological scenarios in augmented reality (AR): Results of different experiments.Applied Sciences, 9(14), 1-16.http://dx.doi.org/ 10.3390/app9142907

Castañeda, L., Esteve, F., \&Adell, J. (2018). ¿Por qué es necesario repensar la competencia docente para el mundo digital? RED. Revista de Educación a Distancia, 56, 1-20. http://dx.doi.org/ $10.6018 / \mathrm{red} / 56 / 6$.

Chen, P., Liu, X., Cheng, W., \& Huang, R. (2017). A review of using Augmented Reality in Education from 2011 to 2016. In Popescu et al. (Eds), Innovations in Smart Learning (pp. 1318). Singapore: Springer.

Cheng, K. H. (2017). Reading an augmented reality book: An exploration of learners' cognitive load, motivation, and attitudes. Australasian Journal of Educational Technology, 33(4), 53-69. https://doi.org/10.14742/ajet.2820

Delello, J. A. (2014). Insights from pre-service teachers using science-based augmented reality. Journal of computers in education, 1(4), 295-311. doi: 10.1007/s40692-014-0021-y

Díaz, J., Molina-García, J., \& Monfort-Pañego, M.(2019). Estudio de las actitudes y el interés de los docentes de primaria de educación física por las TIC en la Comunidad Valenciana. Retos: nuevas tendencias en educación física, deporteyrecreación,(35), 267-272.

Engels, E. S., \& Freund P. A. (2019). Entwicklung und Validierungeines Fragebogens zur Erfassungvon Freude am Schulsportim Jugendalter (FEFS-J). Diagnostica, 65, 166178. doi: $10.1026 / 0012-1924 / \mathrm{a} 000222$

Fombona,J., Pascual, M.Á., \& González, M.C.(2017).M-learning y realidad aumentada: Revisión de literatura científica en el repositorio WoS. Comunicar, 25(52), 63-72.https://doi.org/ 10.3916/C52-2017-06

Fombona, J., \& Pascual, M. Á. (2017). La producción científica sobre Realidad Aumentada, un análisis de la situación educativa desde la perspectiva SCOPUS. EDMETIC, 6(1), 3961.

Fombona,J., \& Vázquez, E. (2017). Posibilidades de utilización de la Geolocalización y RealidadAumentada en el ámbito educativo. Educación XX1, 20(2), 319-342. http://dx.doi.org/ 10.5944/educXX1.10852

Fuentes, A., López, J., \& Pozo, S. (2019). Analysis of the Digital Teaching Competence: Key Factor in the Performance of Active Pedagogies with Augmented Reality. REICE. Revis- 
ta Iberoamericana sobre calidad, eficacia y cambio en educación, 17(2),27-42. doi: 10.15366/reice2019.17.2.002

Gallego-Lema, V., Muñoz-Cristobal,J.A.,Arribas-Cubero,H.F., \& Rubia-Avi, B. (2017). Orienteering in the natural environment: ubiquitous learning through the use of technology. Movimento, 23(2), 755-770.

Garay, U., Tejada, E., \& Castaño, C. (2017). Percepciones del alumnado hacia el aprendizaje mediante objetos educativos enriquecidos con realidad aumentada. EDMETIC, 6(1), 145 164.

Gómez-Gonzalvo, F., Molina-Alventosa, J.P., \& Devís, J. (2018). Los videojuegos como materiales curriculares: una aproximación a su uso en Educación Física. Retos: nuevas tendencias en educación física, deporte y recreación, (34), $305-$ 310.

Gómez,M., Trujillo,J.M.,Aznar,I., \& Cáceres,M.P.(2018).Augment reality and virtual reality for the improvement of spatial competences in Physical Education. Journal of Human Sport and Exercise, 13(2proc), 189-198.

Heradio, R., de la Torre,L., Galán, D., Cabrerizo,F.J.,Herrera, E., \& Dormido, S. (2016). Virtual and remote labs in education: A bibliometric analysis. Computers \& Education, 98, 14-38. doi: 10.1016/j.compedu.2016.03.010

Hernández, R., Fernández, C., \& Baptista, M. P.(2014). Metodología de la investigación. Madrid: McGraw Hill.

Hsiao, K.F. (2013). Using augmented reality for students health case of combining educational learning with standard fitness. Multimedia tools and applications, 64(2), 407-421. doi: 10.1007/s11042-011-0985-9

Huang, Y.J., \& Reynoso, L.C. (2018). Based on Physical SelfConcept to Discuss the Effect of Environmental Education on Health Related Physical Education. EKOLOJI, 27, 1645 1651.

Jaramillo,A. M., Silva, G. J.,Adarve, C.A., Velásquez, S. M., Páramo, C.A., \& Gómez, L. L. (2018). Aplicaciones de Realidad Aumentada en educación para mejorar los procesos de enseñanza-aprendizaje: una revisión sistemática. Revista Espacios, 39(49), 1-15.

López, J., Pozo, S., \& López, G. (2019). La eficacia de la realidad aumentada en las aulas de infantil: un estudio del aprendizaje de SVB y RCP en discentes de 5 años. Pixel-Bit. Revista de Mediosy Educación, 55, 157-178. https://doi.org/10.12795/ pixelbit.2019.i55.09

Lohbeck, A., Engels, E.S., \& Freund, P.A. (2019). Assessing Students` Enjoyment in Physical Education: Measurement invariance across school tracks and relationships with grades. Journal of Psychoeducational Assessment, 37(8), 1023-1029. doi: $10.1177 / 0734282918804600$

Lorenzo, G., \& Scagliarini, C. (2018). Bibliometricreview of augmentedreality in education. Revista general de informaciónydocumentación, 28(1),45-60. doi: 10.5209/RGID.60805

Marín, V., Cabero, J., \& Gallego, O. M. (2018). Motivación y realidad aumentada: Alumnos como consumidores y productores de objetos de aprendizaje. Aula Abierta, 47(3), 337-346.

Marín, V., \& Muñoz, V.P. (2018). Trabajar el cuerpo humano con realidad aumentada en educación infantil. Revista Tecnología, Ciencia y Educación, (9), 148-158.

Marqués, L., Cela, J., \& Gisbert, M. (2017). Pre-service Physical Education Teachers' self-management ability: a training experience in 3D simulation environments. Retos: nuevas tendencias en educación física, deporte y recreación, (32), 30-34.

Milton, D.,Appleton, P.R., Bryant,A., \& Duda, J.L. (2018). Initial Validation of the Teacher-Created Empowering and Disempowering Motivational Climate Questionnaire in Psysical Education. Journal of Teaching in Physical Education,37(4),340-351.doi: 10.1123/jtpe.2018-0119

Muhamedyev, R.I., Aliguliyev, R.M., Shokishalov, Z.M., \& Mustakayev, R.R. (2018). New bibliometric indicators for prospectivity estimation of research fields, 65(1), 1-8.

Muñoz-Cristobal, J.A., Gallego-Lema, V., Arribas-Cubero, H.F., Martínez-Mones, A., \&Asensio-Pérez, J.I. (2017). Using virtual learning environments in bricolage mode for orchestrating learning situations across physical and virtual spaces. Computers \& Education, 109, 233-252. doi: 10.1016/ j.compedu.2017.03.004

Opris, I., Costinas, S., Ionescu, C.S., \& Gogoase, D.E. (2018). Step-by-step augmented reality in power engineering education. Computersapplications in engineeringeducation, 26(5), 1590-1602. doi: 10.1002/ cae. 21969

Prendes, C. (2015). Realidad aumentada y educación: análisis de experiencias prácticas. Pixel-Bit. Revista de Medios y Educación, 46, 187-203.

Radu, I. (2014). Augmentedreality in education: a meta-review and cross-media analysis. Personal and Ubiquitous Computing, 18(6), 1533-1543.

Rodríguez,A. M., Cáceres, M.P., \&Alonso, S. (2018). La competencia digital del futuro docente: análisis bibliométrico de la productividad científica indexada en Scopus. International Journal of Educational Research and Innovation. IJERI, 10,317-333.

Rodríguez, A. M., Hinojo, F. J., \& Ágreda, M. (2019). Diseño e implementación de una experiencia para trabajar la interculturalidad en Educación Infantil a través de realidad aumentada y códigos QR. Educar, 55(1), 59-77. https:// doi.org/10.5565/rev/educar.966

Roig-Vila, R., Lorenzo-Lledó,A., \& Mengual-Andres, S. (2019). Perceived usefulness of augmented reality as a didactic resource in the Infant Education Teacher Degree. Campus Virtuales, 8(1), 19-35.

Ruíz-Ariza,A., Casuso, R.A., Suárez-Manzano, S., \& MartínezLópez, E.J.(2018). Effect of augmented reality game Pokemon $\mathrm{GO}$ on cognitive performance and emotional intelligence in adolescent young. Computers \& Education, 116, 49-63. doi: 10.1016/j.compedu.2017.09.002

Suh,A., \& Prophet, J. (2018). The state of immersive technology research: A literature analysis. Computers in human behaviour, 86(77), 77-90. doi: 10.1016/j.chb.2018.04.019

Toledo, P., \& Sánchez, J. M. (2017). RealidadAumentada enEducación Primaria: efectos sobre el aprendizaje. RELATEC: Revista Latinoamericana de Tecnología Educativa, 16(1), 79-92.

Villalustre,L., \& del Moral, M.E. (2017). Juegos perceptivos con realidad aumentada para trabajar contenido científico. Educação, Formação \& Tecnologias, 10(1), 3646.

Viñals, A., \& Cuenca, J. (2016). El rol del docente en la era digital. Revista Interuniversitaria de Formación del Profesorado, 30(2), 103-114. 